\title{
Voluntary contributions with imperfect information: An experimental study
}

\author{
M. Vittoria Levati · Andrea Morone · Annamaria Fiore
}

Received: 30 May 2007 / Accepted: 16 July 2008 / Published online: 12 August 2008

(C) The Author(s) 2008. This article is published with open access at Springerlink.com

\begin{abstract}
This paper uses a two-person linear voluntary contribution mechanism with stochastic marginal benefits from a public good to examine the effect of imperfect information on contributions. Estimates of individual risk preferences are obtained using data from second-price auctions over lotteries. The results show that limited information about the value of the public good significantly lowers average contributions in all periods but the last. Moreover, the results support the interpretation that subjects bid "as if" they were risk averse, and suggest that "as if" risk-averse behavior is negatively correlated with willingness to contribute.
\end{abstract}

Keywords Public goods experiments · Second-price auctions · Imperfect information · Risk

JEL Classification $\mathrm{C} 72 \cdot \mathrm{C} 92 \cdot \mathrm{D} 80 \cdot \mathrm{H} 41$

\section{Introduction}

A significant body of literature on linear public goods games has accumulated in the last 20 years. One reason for this enduring interest is that, although such games are usually parameterized to create a social dilemma (Dawes 1980), a large percentage of participants in public goods experiments are found to voluntarily cooperate. In repeated settings, however, contributions decline over time and reach their minimum when the interaction terminates (see Ledyard 1995 for a review). These findings have stimulated research aimed at explaining the observed behavior and ascertaining features of the institution or environment that

M.V. Levati $(\bowtie)$

Max Planck Institute of Economics, 07745 Jena, Germany

e-mail: levati@econ.mpg.de

M.V. Levati · A. Morone · A. Fiore

Dipartimento di Scienze Economiche e Metodi Matematici, University of Bari, Bari, Italy 
may influence it. This paper focuses on two related features: the information conveyed to the agents and their "as if" risk preferences. ${ }^{1}$

Most previous experimental studies have been performed in a rich informational environment in which the public good's value was common knowledge. Yet, in real life, a person hardly knows in advance the marginal benefits she can derive from a public good she is asked to finance. Public goods normally are supplied after contributions are made, and thus initial contributors do not know a priori the value of the good. The question can therefore be raised as to whether or not the voluntary cooperation typically observed in linear contribution mechanisms would endure in a world of imperfect information about the public good's value.

The experiment reported here is designed to address this question. A linear voluntary contribution mechanism is tested under two informational environments. Under perfect information (henceforth, PI-treatment), the marginal value of the public good is certain and known to all. This condition is similar to those used in previous studies. Under imperfect information (henceforth, II-treatment), the marginal benefit from the public good can take one of two (equiprobable) values, and each subject knows these values and their probability distribution.

By implementing two information conditions this paper is connected to a (small) strand of experimental literature that considers the effect of incomplete information on contributions. In an asymmetric step level public goods experiment, van Dijk and Grodzka (1992) find no difference in contribution levels between subjects who know only their own endowment and subjects who also know the endowments of the others. In a similar vein, van Dijk et al. (1999) find that the overall (group) averages in their complete and partial information conditions are very similar. Marks and Croson (1999) analyze a provision point game where subjects have incomplete information about the valuations of others, and report no significant difference in the level of group contributions between information conditions. ${ }^{2}$ All these experiments assume private information. This paper moves a step further and supposes that one does not know her own marginal benefit from the public good, but she is informed that it can take one of two values with the same probability.

The stochastic value adds an element of risk to the standard public goods setting, thereby allowing one to explore whether, and to what extent, the presence of risk affects the usually observed behavioral patterns. To make the two treatments comparable, the parameters are chosen such that the Nash equilibrium and the efficient outcome are corner solutions, opposite to each other, both when the information is perfect and when it is imperfect and participants are risk-neutral. Thus, under risk-neutrality, behavior should not differ between treatments.

\footnotetext{
${ }^{1}$ The phrase "as if" is used to acknowledge that experimenters cannot actually observe the subjects' preference characteristics. They can only estimate values for their risk preference parameters using, sometimes, specific expected utility models. This work does not claim that the calculated parameter values capture 'true' preferences or that a particular specification of the utility function is the only possible representation of risky choice. With readers' understanding, in the rest of the paper, behavior consistent with risk aversion (risk loving) will be referred to as risk-averse (risk-loving) behavior.

${ }^{2}$ On theoretical grounds, it is worth mentioning the studies by Gradstein et al. (1994) and Menezes et al. (2001). Gradstein et al. investigate the robustness of the neutrality theorem when an individual does not know the others' income, and find that attaining neutrality is more difficult with incomplete information. Menezes et al. confirm the superiority of subscription games over contribution games in the presence of incomplete information about the others' valuations of the public good.
} 
However, Bagnoli and Lipman (1989, p. 585) suggest that a lack of information, as created in the $I I$-treatment, may lower individuals' willingness to contribute. ${ }^{3}$ Furthermore, Ledyard (1995, p. 143) lists risk aversion among the systematic variables which may shape contributions levels, and acknowledges that its effect has not been tested. To the best of our knowledge, the relation between "as if" risk attitudes and willingness to contribute has remained largely unexplored, although there is some literature that comes close to the issue. For instance, in a non-linear public goods game involving risk about one's own and/or another person's marginal benefit from the public good, Brennan et al. (2008) find that contributions are, on average, decreasing in both the own and the other's risk. ${ }^{4}$ Walker and Halloran (2004) investigate a one shot voluntary contribution mechanism in which the imposition of rewards or sanctions (a "nested" public good) is uncertain. Their study reveals that uncertainty about reward or sanction does not alter behavior as compared to a setting with certain rewards and sanctions and to a baseline treatment with no sanction and no reward.

Due to the crucial role of risk preferences in our setup, it is important to estimate values for subjects' risk preference parameters. To do so, individual valuations of ten risky prospects are elicited using an incentive-compatible second-price auction prior to the public goods game. ${ }^{5}$ Valuations are defined as reservation prices that a person is willing to pay to buy the prospect.

Section 2 provides details of the experimental procedures and treatments. Section 3 reports the results of the experiment. Section 4 summarizes our central findings and concludes.

\section{The experiment}

The computerized experiment was performed at the laboratory of the Max Planck Institute in Jena (Germany). The experiment was programmed using the z-Tree software (Fischbacher 2007). Participants were undergraduate students from different disciplines at the University of Jena.

In total, two experimental sessions were run, each involving 32 participants, and implementing one of the two treatments (between-subjects design). Each session consisted of two different experiments. First, subjects participated in a series of second-price auctions. They then played a repeated linear public goods game under either perfect or imperfect information. The instructions distributed at the beginning informed participants that they would take part in two separate experiments, and explained the rules of the first experiment only. Written instructions on the second experiment were distributed at the end of the first one (a translation of the German instructions for the two experiments is available upon request). In both experiments amounts were denoted by ECU (Experimental Currency Unit), where

\footnotetext{
${ }^{3}$ Actually, Bagnoli and Lipman have in mind a voluntary contribution mechanism for the provision of threshold public goods.

${ }^{4}$ Brennan et al.'s paper differs from the current study in that it examines the relationship between otherregarding concerns and attitudes toward "social" risk in experiments involving choices with and without strategic interaction.

${ }^{5}$ The second-price auction is a market institution that should induce subjects to reveal their true values (for examples of its application see Shogren et al. 1994; Di Mauro and Maffioletti 2004). Other approaches to measuring risk preference parameters are the Becker-DeGroot-Marschak mechanism, the binary lottery technique, and the paired lottery-choice task.
} 
Table 1 Experimental lotteries

\begin{tabular}{|c|c|c|c|c|c|c|}
\hline \multirow{10}{*}{ Table 1 Experimental lotteries } & \multirow{2}{*}{\multicolumn{3}{|c|}{$\begin{array}{l}P I \text {-treatment } \\
L=75 \mathrm{H}=175\end{array}$}} & \multirow{2}{*}{\multicolumn{3}{|c|}{$\begin{array}{l}I I \text {-treatment } \\
L=40 H=220\end{array}$}} \\
\hline & & & & & & \\
\hline & $\bar{\theta}$ & EV & $\sigma^{2}$ & $\theta$ & $\mathrm{EV}$ & $\sigma^{2}$ \\
\hline & 0.8 & 95 & 1600 & 0.55 & 121 & 8019 \\
\hline & 0.5 & 125 & 2500 & 0.7 & 94 & 6804 \\
\hline & 0.55 & 120 & 2475 & 0.55 & 121 & 8019 \\
\hline & 0.6 & 115 & 2400 & 0.6 & 112 & 7776 \\
\hline & 0.65 & 110 & 2275 & 0.65 & 103 & 7371 \\
\hline & 0.7 & 105 & 2100 & 0.7 & 94 & 6804 \\
\hline & 0.75 & 100 & 1875 & 0.75 & 85 & 6075 \\
\hline \multirow{3}{*}{$\begin{array}{l}\text { Note: } \mathrm{EV} \text { and } \sigma^{2} \text { denote, } \\
\text { respectively, expected value and } \\
\text { variance. } \theta \text { is the probability of } \\
\text { the low outcome } L\end{array}$} & 0.8 & 95 & 1600 & 0.8 & 76 & 5184 \\
\hline & 0.85 & 90 & 1275 & 0.85 & 67 & 4131 \\
\hline & 0.9 & 85 & 900 & 0.9 & 58 & 2916 \\
\hline
\end{tabular}

Note: $\mathrm{EV}$ and $\sigma^{2}$ denote, respectively, expected value and variance. $\theta$ is the probability of the low outcome $L$

$10 \mathrm{ECU}=€ 1$. In order to avoid portfolio-diversification effects, participants in each session/treatment were paid according to one choice only. ${ }^{6}$ The average payoff, earned in about $1 \frac{1}{2}$ hours, was $€ 11.7$ (including a show-up fee of $€ 2.50$ ).

\subsection{The second-price auctions}

The first experiment collects data from a series of second-price auctions so as to estimate individual risk preference parameters. Each participant submits the maximum integer amount she is willing to pay to acquire a lottery yielding a payoff of $L$ or $H(0<L<H)$ with probabilities $\theta$ and $1-\theta$, respectively. Each player must bid on 10 lotteries, being aware that the right to play each lottery is granted to the highest bidder at a price equal to the second highest bid. Table 1 lists the set of implemented lotteries separately for each treatment. To simplify the task, the probabilities attached to the two possible payoffs are presented not only numerically but also graphically in the form of pie charts.

The low (high) payoff $L(H)$ attainable in each lottery-set is chosen so as to equalize the minimum (maximum) payoff achievable in the subsequent public goods game. In this way, risk preferences refer always to the same expected wealth. This is important as risk attitudes may vary with wealth (Rabin and Thaler 2001).

For each auction, participants receive an endowment of 100 ECU. Yet, due to some expected values exceeding 100 (see Table 1), participants are informed that bids can be higher than their own endowment, and thus paid out of their own pockets. ${ }^{7}$ No feedback about the ten lotteries is provided until the end of the experimental session. Only after playing the public goods game is each subject informed of each lottery's highest and second highest bid as well as of whether or not she wins that lottery. If one of the lotteries is randomly chosen for payment, the subject who submitted the highest bid pays the second highest bid and acquires the right to play out that lottery so that her payments are contingent on the

\footnotetext{
${ }^{6}$ The following random lottery incentive system was used. First, one of the two experiments was selected at random, with both experiments being equally likely. Then, depending on whether the random device selected the first or second experiment, either one of the 10 lotteries or one of the 10 periods was randomly picked up, where all lotteries/periods were equally likely.

${ }^{7}$ Note that subjects can actually experience losses only if their bids exceed 125 (i.e., endowment plus participation fee) plus the worse outcome of the lottery.
} 
lottery's outcome. All other subjects earn their initial endowment. Under the assumption of separability (implying that each auction is seen as an independent event), this random lottery incentive mechanism gives subjects the appropriate motivation to reveal their true preferences in each auction. ${ }^{8}$

\subsection{The public goods game}

The second experiment investigates the impact of imperfect information on contributions. To achieve this aim, it relies on the standard linear public goods game as introduced by Isaac et al. (1984).

Groups of size two interact for 10 periods in a partners design. ${ }^{9}$ In any one period, each participant is endowed with $100 \mathrm{ECU}$ and must decide privately how much to contribute to a public good, keeping the remaining ECU for herself. Let $c_{i}$ denote individual $i$ 's contribution to the public good (with $c_{i}=\{1,2,3, \ldots, 100\}, i=1,2$ ), and let $C=c_{1}+c_{2}$ be the total amount of public good provided. The monetary payoff of each $i$ is given by

$$
U_{i}\left(c_{i}, C\right)=100-c_{i}+\alpha C,
$$

where $\alpha$ is $i$ 's marginal benefit from the public good.

In the PI-treatment, subjects are told that $\alpha$ is fixed at 0.75 ; this is the control treatment. ${ }^{10}$ In the $I I$-treatment, subjects are informed that $\alpha$ can be either $\underline{a}=0.4$ or $\bar{a}=1.1$, with probability $1 / 2$ each. Notice that the expected marginal benefit from the public good is kept constant across treatments to focus solely on the effects of imperfect information, although the two values of $\alpha$ in the $I I$-treatment (and, thus, the parameterized marginal payoffs) provide the players with opposite incentives for (not) contributing.

To avoid confounding effects due to asymmetric marginal benefits, the value of $\alpha$ is simultaneously determined for both members of a pair, and subjects know this. In both treatments, at the end of each period, participants get feedback on their partner's contribution and their private payoff. In the II-treatment, subjects are also informed about the randomly chosen $\alpha$-value in their own pair.

A risk-neutral player facing our parameters should contribute zero in both treatments if she is strictly self-interested, while she should always fully contribute if she is efficiencyoriented (i.e., wants to maximize the sum of $U_{1}$ and $U_{2}$ ). However, in the $I I$-treatment, the induced (unobserved) marginal benefit, $\alpha_{i}$, to risk-averse or risk-loving agent $i$ may be such

\footnotetext{
${ }^{8}$ We acknowledge Holt's (1986) and Karni \& Safra's (1987) critique according to which subjects consider the whole experiment as a single decision problem, thereby choosing a set of optimal answers given their preference functional. However, in the setting under investigation, such a behavior would require a high degree of sophistication. Additionally, Cubitt et al. (1998) show that the random lottery incentive mechanism does work properly.

${ }^{9}$ It was desired to make the two treatments equivalent under risk-neutrality and, at the same time, to have $\alpha$-parameters inducing opposite material incentives in the $I I$-treatment. Groups of minimal size reach these objectives without departing too much from the typical marginal efficiency gains (of 1.6) attainable through cooperation in case of perfect information. Had the design considered, for instance, four-person game, the $\alpha$-parameters in II could not have been between 0.3 and 1 to yield opposite predictions. However, values of, e.g., 0.1 and 1.1 in $I I$ would have implied a marginal return of 0.6 per person (a total of 2.4 for the group) in PI. It is well-known (Rapoport and Chammah 1965) that larger relative payoffs from mutual cooperation compared to mutual defection can affect behavior considerably.

${ }^{10}$ The choice of $\alpha=0.75$ in $P I$ is based on former two-person public goods experiments, such as Coricelli et al. (2004), González et al. (2005), and Staffiero (2006), which rely on marginal values of 0.8, 0.7, and 0.75, respectively.
} 
to eliminate the dilemma. ${ }^{11}$ Let $C E_{i}$ be $i$ 's certainty equivalent for the risky payoff, meaning that receiving $C E_{i}$ for sure is equally preferable to the prospect of receiving either $100-$ $c_{i}+\underline{a} C$ or $100-c_{i}+\bar{a} C$ with probability $1 / 2$ each. The more risk-averse (risk-loving) agent $i$ is, the lower (the higher) will be her $C E_{i}$. In the public goods context under investigation, the certainty equivalent can be expressed as $C E_{i}=100-c_{i}+\alpha_{i} C$. Thus, for given $c_{i}$ and $c_{j}(j=1,2 ; j \neq i)$, a lower/higher $C E_{i}$ implies a lower/higher induced $\alpha_{i}$. If agent $i$ is risk-averse and her $C E_{i}$ corresponds to a payoff with $\alpha_{i}<0.5$, then $i$ should contribute zero both if she is interested only in her own monetary payoff and if she cares about efficiency. Similarly, if $i$ is risk-loving and her $C E_{i}$ corresponds to $\alpha_{i}>1$, then $i$ should contribute her whole endowment if she is either self-interested or efficiency-minded. It is therefore necessary to identify whether the subjects' "as if" risk preference parameters are such to induce $\alpha_{i}$-values that rule out the dilemma in the $I I$-treatment.

Suppose that agent $i$ has a Constant Relative Risk Aversion (CRRA) utility function defined over material payoffs of the form $V_{i}=U_{i}^{r_{i}}$, where $\left(1-r_{i}\right)$ is $i$ 's index of relative risk aversion (see, e.g., Wakker 2008). Then, if agent $i$ in the $I I$-treatment contributes $c_{i}$ to the public good and believes that her partner contributes $b_{i}$, her expected utility is:

$$
V_{i}=\frac{1}{2}\left(100-c_{i}+0.4\left(c_{i}+b_{i}\right)\right)^{r_{i}}+\frac{1}{2}\left(100-c_{i}+1.1\left(c_{i}+b_{i}\right)\right)^{r_{i}} .
$$

Consider now a hypothetical public goods game paying a deterministic payoff equal to the certainty equivalent $C E_{i}$, where $b_{i}$ replaces $c_{j}$. Given $c_{i}$ and $b_{i}, i$ 's utility from this hypothetical public good would be:

$$
V_{i}^{u}=\left(100-c_{i}+\alpha_{i}\left(c_{i}+b_{i}\right)\right)^{r_{i}} .
$$

Setting $V_{i}=V_{i}^{u}$ and solving for $\alpha_{i}$, one gets:

$$
\alpha_{i}=\frac{\left[0.5\left(100-0.6 c_{i}+0.4 b_{i}\right)^{r_{i}}+0.5\left(100+0.1 c_{i}+1.1 b_{i}\right)^{r_{i}}\right]^{\frac{1}{r_{i}}}-100+c_{i}}{c_{i}+b_{i}} .
$$

For specific $c_{i}$ and $b_{i}$, (1) allows an assessment of whether, given her risk preference parameter estimates, subject $i$ considers the public goods game with stochastic marginal benefits as equivalent to a deterministic game with $\alpha_{i} \in(0.5,1)$, thereby confronting the dilemma.

Estimations of $\alpha_{i}$ using (1) require knowing $i$ 's expectations about her partner's contributions. Consequently, in each period of both treatments subjects are asked to predict how many ECUs their partner will contribute in the current period. To encourage subjects to report truthful beliefs, a bonus of $€ 15$ is paid to the participant with the most accurate predictions in each session. ${ }^{12}$

Eliciting expectations about the other's contributions also allows the investigation of whether subjects behave conditionally cooperative, in the sense that they contribute more,

\footnotetext{
${ }^{11}$ One ought to distinguish, in the $I I$-treatment, between the parameterized marginal benefits (namely, 0.4 and 1.1) and the induced marginal benefits to a risk-averse or risk-loving agent, which may lie anywhere between 0.4 and 1.1 .

${ }^{12}$ Previous research in experimental economics has shown that the mere act of eliciting beliefs about the others' actions can affect behavior in finitely repeated public goods games (see, e.g., Croson 2000; Gächter and Renner 2006). As beliefs are elicited in our two treatments, this caveat applies to both of them, thereby leaving unaffected their comparison.
} 
the more others (are expected to) contribute. Several experiments, all performed under perfect information conditions, reveal that conditional cooperation is important (see, e.g., Keser and van Winden 2000; Fischbacher et al. 2001; Levati and Neugebauer 2004). This study allows one to examine whether imperfect information about the public good's value affects beliefs and/or the relationship usually detected between beliefs and actions. ${ }^{13}$

\section{Results}

We start our analysis by classifying subjects according to their risk attitudes, based on their bids in the second-price auctions. We then proceed to examine contribution levels in the two public goods treatments so as to investigate whether and how contributions are affected by the different information conditions. Finally, we examine the relationship between individuals' contribution behavior and our estimates of their risk preference parameters.

\subsection{Risk attitude estimates}

To assess each subject's "as if" risk attitudes, we calculate her local absolute risk aversion coefficient, as originally proposed by Pratt (1964). The advantage of this method is that it does not rely on a specific functional form for the utility function.

Define $\pi(x, \tilde{z})$ to be the risk premium a decision maker is willing to pay out of her nonstochastic wealth $x$ to avoid a risky prospect $\tilde{z}$. Formally, the decision maker is indifferent between $\tilde{z}$ and $E(\tilde{z})-\pi(x, \tilde{z})$, i.e., receiving $\pi(x, \tilde{z})$ less than the expected value of the lottery. If her utility function is $u$, one can write $u(x+E(\tilde{z})-\pi(x, \tilde{z}))=E\{u(x+\tilde{z})\}$. Pratt (1964) shows that expanding $u$ around $x$ on both sides of the latter expression and equalizing the two expansions yields $\rho(x)=\frac{\pi(x, \tilde{z})-o\left(\sigma_{z}^{2}\right)}{0.5 \sigma_{z}^{2}}$, where $\sigma_{z}^{2}$ is the variance of the risky prospect, and, in expansions, $o()$ means "terms of smaller order than".

We use the ratio above to estimate the subject's attitudes to risk. Omitting $o\left(\sigma_{z}^{2}\right)$, we can easily assess the variance $\sigma_{z}^{2}$ of each lottery and the risk premium $\pi(x, \tilde{z})$. In particular, for each lottery $\tilde{z}$ and each subject, $\pi(x, \tilde{z})$ is calculated as the difference between the expected value of $\tilde{z}$ and the subject's elicited willingness to pay for $\tilde{z}$. A coefficient $\rho$ equal to 0 indicates risk neutrality, whereas a coefficient greater (smaller) than zero is suggestive of risk aversion (proneness). Tables 2 and 3 list the average (over the 10 lotteries) risk aversion coefficient $\rho$ of each individual subject for the $P I$ - and the $I I$-treatment, respectively.

Thirty (29) out of 32 participants in the $I I$ - (PI-)treatment behave as risk-averse economic agents; the remaining 2 (3) participants appear risk-loving. In sum, the following result corroborates earlier experimental evidence (see, e.g., Holt and Laury 2002; Dohmen et al. 2006):

Result 1 In both sessions/treatments, more than 90\% of subjects bid as if they were riskaverse.

Our next step is to use (1) to infer the induced marginal benefits to participants in the $I I$ treatment. To do so, we need to estimate the numerical values of $r_{i}$ using certainty equivalent data. As subjects' responses are typically affected by errors (Hey and Orme 1994), we add

\footnotetext{
${ }^{13}$ Previous experimental studies measuring the correlation between beliefs and contributions include González et al. (2005), Fischbacher and Gächter (2006), and Neugebauer et al. (2008).
} 
Table 2 Individual estimates of $\rho$ in the $P I$-treatment (estimations based on Arrow-Pratt measure)
Table 3 Individual estimates of $\rho$ in the $I I$-treatment (estimations based on Arrow-Pratt measure)

\begin{tabular}{llll}
\hline Subject & $\rho$ & Subject & $\rho$ \\
\hline 1 & 0.05374 & 17 & 0.03530 \\
2 & 0.01380 & 18 & 0.10693 \\
3 & -0.03722 & 19 & 0.06754 \\
4 & 0.05699 & 20 & 0.01657 \\
5 & 0.04993 & 21 & 0.03982 \\
6 & 0.00429 & 22 & 0.03790 \\
7 & 0.05814 & 23 & 0.05548 \\
8 & 0.05740 & 24 & 0.03415 \\
9 & 0.07223 & 25 & 0.10766 \\
10 & -0.00089 & 26 & 0.11637 \\
11 & 0.05462 & 27 & 0.08490 \\
12 & 0.06553 & 28 & 0.10830 \\
13 & 0.05840 & 29 & 0.03399 \\
14 & -0.00332 & 30 & 0.05562 \\
15 & 0.02759 & 31 & 0.10675 \\
16 & 0.08148 & 32 & 0.02915
\end{tabular}

\begin{tabular}{llll}
\hline Subject & $\rho$ & Subject & $\rho$ \\
\hline 1 & 0.02479 & 17 & 0.01817 \\
2 & 0.02237 & 18 & 0.01455 \\
3 & 0.00936 & 19 & 0.01979 \\
4 & 0.01865 & 20 & 0.01834 \\
5 & 0.01940 & 21 & 0.01591 \\
6 & 0.00701 & 22 & 0.01538 \\
7 & 0.01320 & 23 & 0.01650 \\
8 & 0.02102 & 24 & 0.01465 \\
9 & 0.01947 & 25 & 0.02216 \\
10 & 0.01980 & 26 & 0.01657 \\
11 & -0.00375 & 27 & 0.01610 \\
12 & 0.01592 & 28 & 0.01619 \\
13 & 0.01397 & 29 & 0.00919 \\
14 & 0.01741 & 30 & 0.01756 \\
15 & 0.01617 & 31 & 0.01584 \\
16 & -0.00473 & 32 & 0.02170 \\
\hline
\end{tabular}

a normally distributed (with mean 0 and variance 1) error term to the CRRA utility function $\left(V_{i}^{*}=V_{i}+\varepsilon_{i}\right)$. To derive the estimates of the relative risk aversion coefficient $\left(1-r_{i}\right)$, we use maximum likelihood methods. ${ }^{14}$

\footnotetext{
${ }^{14}$ See Hey and Orme (1994), Hey et al. (2006), and Morone and Schmidt (2008) for details about this estimation technique. 
Table 4 Individual estimates of $r_{i}$ and $\alpha_{i}$ in the $I I$-treatment (estimations via maximum likelihood)

\begin{tabular}{clllll}
\hline Subject & $r_{i}$ & $\alpha_{i}$ & Subject & $r_{i}$ & $\alpha_{i}$ \\
\hline 1 & 1.053 & 0.748 & 17 & 0.729 & 0.738 \\
2 & 0.949 & 0.745 & 18 & 0.999 & 0.750 \\
3 & 0.856 & 0.744 & 19 & 0.736 & 0.746 \\
4 & 0.629 & 0.734 & 20 & 0.677 & 0.745 \\
5 & 0.826 & 0.736 & 21 & 0.890 & 0.748 \\
6 & 0.758 & 0.733 & 22 & 0.632 & 0.744 \\
7 & 0.923 & - & 23 & 0.723 & 0.731 \\
8 & 0.798 & 0.748 & 24 & 0.831 & 0.737 \\
9 & 0.954 & 0.749 & 25 & 0.603 & 0.726 \\
10 & 0.731 & 0.747 & 26 & 0.651 & 0.730 \\
11 & 1.093 & 0.750 & 27 & 0.731 & 0.746 \\
12 & 0.997 & 0.750 & 28 & 0.714 & 0.745 \\
13 & 0.676 & 0.744 & 29 & 0.656 & 0.724 \\
14 & 0.781 & 0.748 & 30 & 0.659 & 0.722 \\
15 & 0.910 & 0.745 & 31 & 0.753 & - \\
16 & 1.290 & 0.750 & 32 & 0.670 & 0.748 \\
\hline
\end{tabular}

Table 4 reports individual estimates of $r_{i}$ and $\alpha_{i}$ using data from the $I I$-treatment. ${ }^{15}$ Two subjects' $\alpha_{i}$ cannot be computed because their contributions $\left(c_{i}\right)$ and their beliefs about the other's contributions $\left(b_{i}\right)$ are equal to zero in all periods. The estimated $r_{i}$ of the remaining 30 participants imply (given their $c_{i}$ and their $b_{i}$ ) $\alpha_{i}$-values between 0.72 and 0.75 . We can therefore state:

Result 2 In the II-treatment, almost 94\% of the subjects seem to consider the stochastic public goods game as equivalent to a hypothetical public goods game with deterministic marginal benefits close to 0.75 . Hence, they face the standard conflict between their own and the group's interest.

Before turning to the analysis of contribution behavior, we examine the "consistency" of individual decisions across lotteries by focusing on the decision maker's certainty-equivalent ratios (CER), i.e., her willingness to pay for a lottery divided by that lottery's expected value (see, e.g., Kachelmeier and Shehata 1992, 1994; Shupp and Williams 2008). ${ }^{16}$ A ratio equal to unity indicates risk neutrality; a ratio lower (greater) than unity suggests risk aversion (seeking). Figures 1 and 2 display the CER mean and median for the two treatments in each of the 10 lotteries; the latter are ordered on the $x$-axis from the least to the most risky.

In both treatments and for both measures used, the ratios are systematically below 1 , indicating that, using either mean or median CERs, the subjects appear consistently riskaverse. Moreover, there is a downward trend in both the mean and the median CER as the risk increases, even though the pattern is not monotonic. A closer look at the individual data reveals that the variance in individual decisions across lotteries is rather high. This finding is consistent with that of, e.g., James (2007), who estimates risk preference parameters on

\footnotetext{
${ }^{15}$ The estimations in Table 4 mirror qualitatively those in Table 3 . Although in Table 4 participant 1 is classified as risk-loving, her $r_{i}$-coefficient is the closest to one.

${ }^{16}$ We thank the referee who requested that we explore this issue.
} 
PI-Treatment

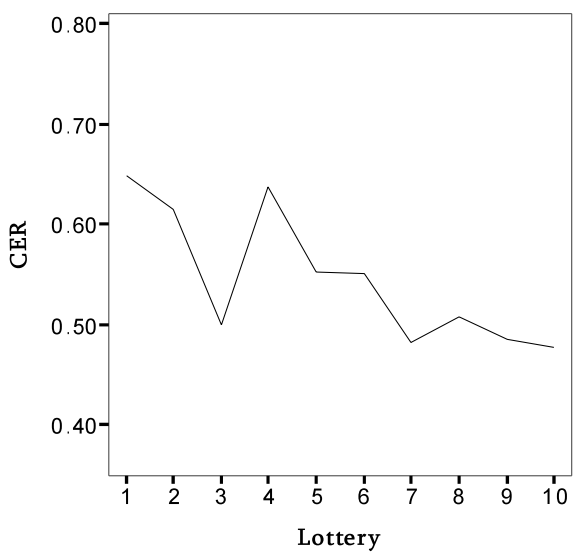

II-Treatment

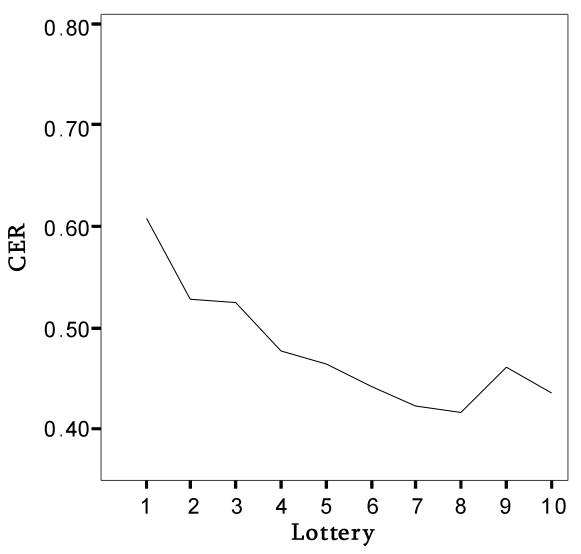

Fig. 1 Mean certainty-equivalent ratio in each of the 10 lotteries (ordered from the least to the most risky), separately for the $P I$ - and the $I I$-treatment

PI-Treatment

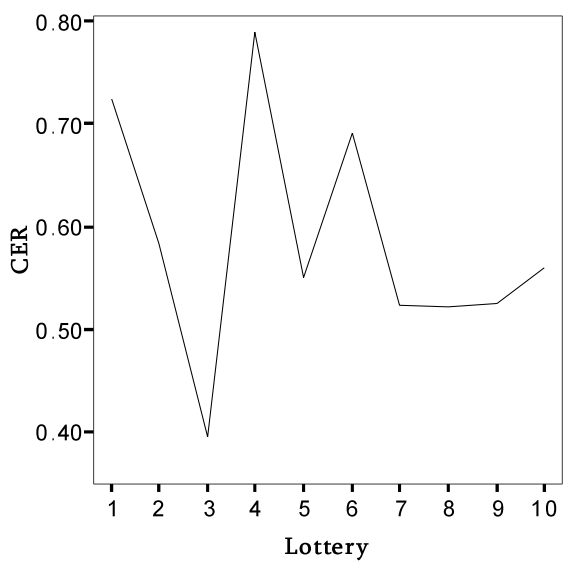

II-Treatment

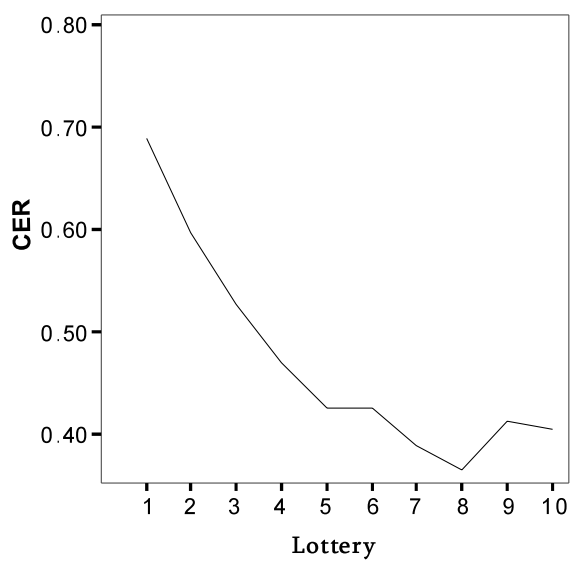

Fig. 2 Median certainty-equivalent ratio in each of the 10 lotteries (ordered from the least to the most risky), separately for the $P I$ - and the $I I$-treatment

a time series of data from the Becker-DeGroot-Marschak mechanism, breaking the series into four different regimes. According to his estimates, most subjects switch from risk aversion to risk seeking behavior (or vice versa) in moving from one treatment regime to the other. ${ }^{17}$ James explains this result by "choice error" and confusion on the part of the subjects. Interestingly, in our experiment, individual CERs - though volatile — are always below

\footnotetext{
${ }^{17}$ Studies documenting risk preference parameter inconsistency across institutions, rather than within one institution, are Isaac and James (2000) and Berg et al. (2005).
} 
Table 5 Summary statistics on contributions in the two treatments

\begin{tabular}{lccccc}
\hline & Mean & Median & Std. dev. & $\%$ of $c_{i}=0$ & $\%$ of $c_{i}=100$ \\
\hline$P I$-treatment & 78.08 & 100 & 35.33 & 9.06 & 65.94 \\
$I I$-treatment & 40.75 & 40 & 35.30 & 25.31 & 12.81 \\
\hline
\end{tabular}
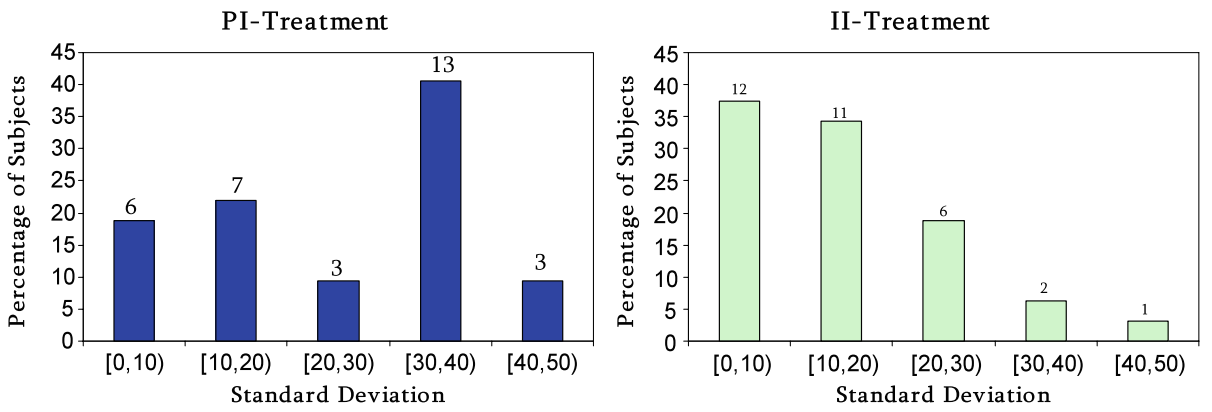

Fig. 3 Standard deviations of contributions of individual subjects across periods in each treatment (the number on top of bars is the total number of subjects in the interval)

1 for $(69 \%) 88 \%$ of the participants in the $(P I-) I I$-treatment, thereby suggesting that most participants behave as if their risk preferences were consistent.

\subsection{Contribution behavior}

Table 5 summarizes the experimental results under the two public goods treatments. Both the mean and the median contributions differ between the two treatments: as compared to a situation with perfect information, the presence of imperfect information about the public good's value significantly lowers average contributions $(p=0.001$; two-sided Wilcoxon rank-sum test; $N=16){ }^{18}$

There is also more full free-riding (contributions of zero) in the $I I$-treatment than in the PI-treatment $(25.31 \%$ versus $9.06 \%$ overall). An exact binomial test rejects the null hypothesis that the proportion of full free-riding is equal in both treatments, in favor of the alternative that the proportion is significantly higher in the $I I$-treatment $(p<0.001)$. A Pearson $\chi^{2}$-test corroborates the robustness of the result $\left(p<0.001, \chi^{2}=15.55\right)$. This evidence gives the first result concerning contribution behavior:

\section{Result 3 Imperfect information significantly decreases average private contributions.}

The average (over the 10 periods) standard deviation of contributions for individual subjects is 22.36 in $P I$ and 15.27 in $I I$. Figure 3 displays the standard deviation of contributions per subject, separately for the two treatments. More than $70 \%$ of the participants in $I I$ have a standard deviation less than 20. Although the corresponding percentage is lower (about

\footnotetext{
${ }^{18}$ Unless otherwise stated, all statistical tests rely on independent group observations. Due to our matching procedure (i.e., partners design), the number of statistically independent groups is $N=16$ for both treatments.
} 


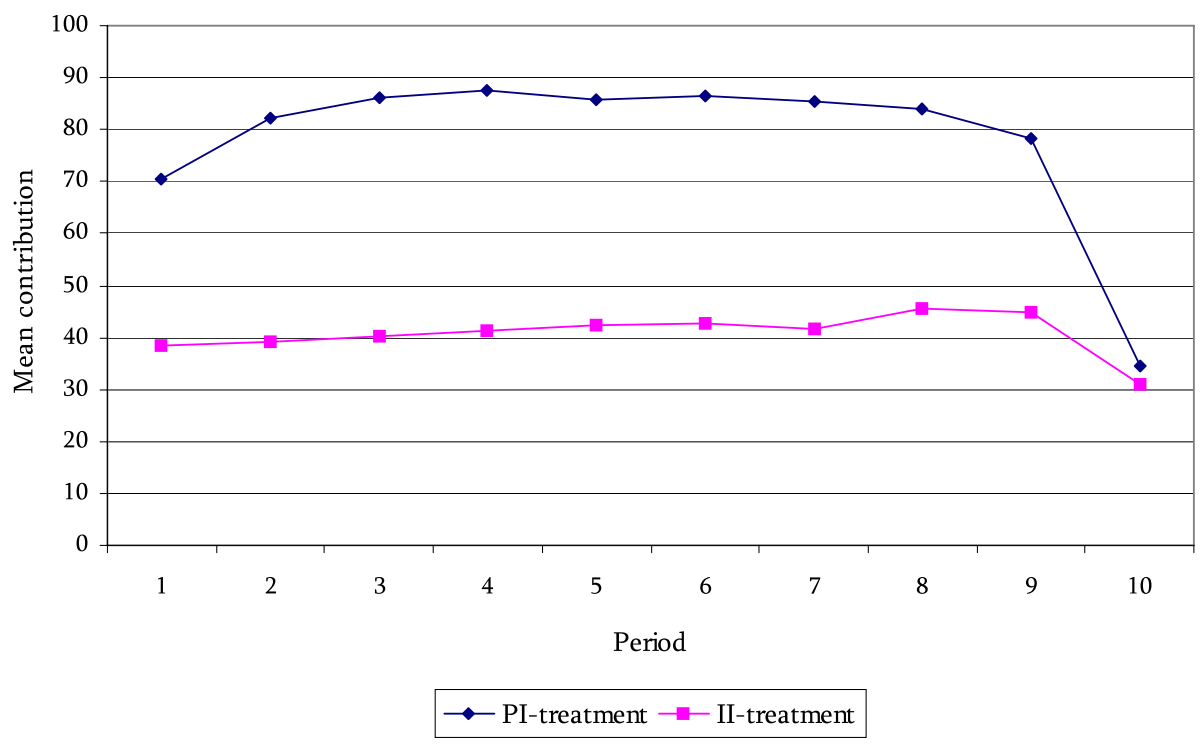

Fig. 4 Average contributions in each period, separately for the $P I$ - and the $I I$-treatment

$40 \%$ ) in $P I$, it increases to $71.8 \%$ when we exclude the last period, where twenty participants $(63 \%)$ decrease their contribution dramatically. ${ }^{19}$ These findings can be summarized as follows:

Result 4 Variation in contributions of individual subjects across periods 1 to 9 is smaller in case of imperfect, rather than perfect, information.

Figure 4 illustrates the time path of average contributions in each treatment. First-period behavior is particularly interesting as it shows how subjects act before receiving any feedback information about their partner (all individual responses are, thus, independent).

Figure 4 reveals that participants in $I I$ start out with significantly lower contributions than participants in $P I$ (a one-sided Wilcoxon rank-sum test delivers $p<0.001 ; N=32$ ). The figure also indicates that average contributions stay roughly constant from the first until the last but one period in both treatments. To support the latter statistically, we performed, separately for each treatment, a generalized linear mixed regression (based on a quasi-Poisson distribution to model over-dispersion) with individual contribution decisions as the dependent variable, and Period (taking values 1 to 10) and the dummy Last Period (which equals 0 for periods 1-9 and 1 for period 10) as independent variables. The model has random effects at two levels: the 16 independent matching groups, and the 320 individual choices. The regression results, reported in Table 6, confirm that there is no time trend in either treatment. ${ }^{20}$

\footnotetext{
${ }^{19}$ Not surprisingly, the variances in contribution between treatments differ significantly $(p=0.013$; twosided Wilcoxon rank-sum test) if the tenth period is included in the sample. However, if period 10 is not counted, the variances in $P I$ and $I I$ are no longer significantly different $(p=0.955)$.

${ }^{20}$ This is at odds with the usual picture of a declining trend (Ledyard 1995) and may depend on our experimental design: interacting finitely often with the same partner may facilitate conditionally cooperative behavior (more on this below).
} 
Table 6 Generalized linear mixed-effects regressions-Individual contributions on periods

\begin{tabular}{lllll}
\hline Independent variable & Coefficient & Std. error & $t$-value & $p$-value \\
\hline PI-treatment & & & & \\
$\quad$ Constant & 4.279 & 0.152 & 28.184 & 0.000 \\
$\quad$ Period & 0.007 & 0.007 & 0.944 & 0.346 \\
$\quad$ Last Period & -0.907 & 0.097 & -9.307 & 0.000 \\
& & & & \\
II-treatment & 3.171 & 0.303 & 10.456 & 0.000 \\
$\quad$ Constant & 0.020 & 0.012 & 1.634 & 0.113 \\
$\quad$ Period & -0.395 & 0.129 & -3.062 & 0.002 \\
$\quad$ Last Period & & & & \\
\hline
\end{tabular}

Moreover, the coefficient of Last Period is always negative and significant, capturing the decline of contributions in the last period of both treatments. ${ }^{21}$ These observations lead to our next result:

Result 5 First-period contributions are significantly lower in case of imperfect information. The time trend of contributions is not different between treatments, though.

Since contribution patterns are qualitatively similar over periods, analyzing behavior in the first period is crucial for shedding light on what triggers the significant quantitative difference between treatments. In both information conditions, first-period individual contributions and first-period individual beliefs are highly significantly correlated: Spearman rank correlation coefficients are 0.83 in $P I$, and 0.80 in $I I(p<0.001$ in each treatment; $N=32$ ). As first-period contributions are significantly lower when information is imperfect, also first-period beliefs are significantly different between treatments ( $p=0.004$; onesided Wilcoxon rank-sum test; $N=32$ ). Based on elicited beliefs about the counterpart's behavior, no subject expected full free-riding in the PI-treatment. In contrast, six subjects had free-riding expectations in the II-treatment.

A possible interpretation of the lower contributions observed in the $I I$-treatment at the outset of the interaction may be that imperfect information affects initial beliefs: when confronted with a risky situation, most participants become pessimistic about their co-player's contribution. A further interpretation is suggested by the "false consensus" hypothesis, according to which people tend to believe that others behave similarly to themselves (Kelley and Stahelski 1970). In this view, expectations of lower contributions may be triggered by one's own predisposition to contribute less. While our experiment is not designed to provide insights into the determinants of beliefs, it clearly shows that a risky payoff has a negative impact on willingness to contribute, and that first-period decisions are critical for future average contribution levels. ${ }^{22}$ A justification for the significant difference in initial behavior between treatments will be provided in the next subsection; it relates to subjects' risk preferences. For the moment, we sum up the evidence on first-period beliefs as follows:

\footnotetext{
${ }^{21}$ One-sided Wilcoxon signed-rank tests comparing averages in the first nine periods and in the last period corroborate the significant end-game effect in both treatments $(p=0.008$ for both treatments; $N=16)$.

${ }^{22}$ The importance of first-period contributions to the whole game has been noticed also by Keser and van Winden (2000), who compare partners and strangers conditions.
} 
Table 7 Generalized linear mixed-effects regression on individual contribution decisions (relying on 640 observations)

\begin{tabular}{lllll}
\hline Independent variable & Coefficient & Std. error & $t$-value & $p$-value \\
\hline Constant & 16.202 & 5.057 & 3.204 & 0.001 \\
Exp $_{i}$ & 0.876 & 0.059 & 14.826 & 0.000 \\
Info & -16.539 & 5.200 & -3.180 & 0.003 \\
Period & 0.927 & 0.679 & 1.366 & 0.172 \\
Last Period & -31.441 & 3.940 & -7.979 & 0.000 \\
Exp $_{i} \times$ Period & -0.024 & 0.007 & -3.357 & 0.001 \\
Info $\times$ Exp $_{i}$ & 0.108 & 0.059 & 1.812 & 0.071 \\
Info $\times$ Period & 0.459 & 0.616 & 0.745 & 0.456 \\
Info $\times$ Last Period & 21.554 & 5.423 & 3.974 & 0.000 \\
\hline
\end{tabular}

Result 6 First-period beliefs regarding the other's contributions are significantly more pessimistic when information is imperfect rather than perfect. In both information conditions, actual and expected contributions in period 1 are highly and positively correlated.

The last result of this subsection investigates whether the relationship between own and expected contributions, detected in the first period, persists over the remaining repetitions of each session/treatment. Before addressing this question, we note that, in all periods following the first, beliefs may become endogenous in the sense of being influenced by the other's observed contributions (see González et al. 2005, and Fischbacher and Gächter 2006, for a thorough discussion of this issue). To check whether this holds true in our experiment, we performed a correlation analysis between beliefs in period $t$ about the partner's contribution in $t$ and the actual contribution of the partner in period $t-1$. The analysis yields significantly positive Spearman's coefficients in both treatments ( 0.92 in $P I, 0.98$ in $I I ; p<0.001$; $N=16$ ). Thus, participants in our experiment anchor their beliefs about the other's contributions on what the other did in the previous period. This is consistent with the work of, e.g., Neugebauer et al. (2008) who observe expectations to depend significantly on the partners' one-period lagged contributions.

To analyze the relationship between beliefs and own contributions, Table 7 reports the results of a generalized linear mixed regression with random effects for matching groups and individuals. The dependent variable is individual $i$ 's contribution decisions $\left(c_{i}\right)$. Regressors are $i$ 's expectations about her partner's contribution in the current period $\left(\operatorname{Exp}_{i}\right)$, a treatment dummy variable (Info) that equals 0 for $P I$ and 1 for the $I I$, and the two variables included in Table 6's regression, i.e., the period index and the dummy for period $10 .^{23}$ The specification of the model includes the interaction of Info with Exp $_{i}$, Period and Last Period as well as the interaction of $\operatorname{Exp}_{i}$ with Period.

Expected contributions have a significantly positive effect on own contributions, especially in the II-treatment (the coefficient of Info $\times \operatorname{Exp}_{i}$ is positive and weakly significant). However, the interaction variable $\operatorname{Exp}_{i} \times$ Period indicates that this positive effect tends to decrease over time. Since beliefs and observed contributions of the partner are highly correlated, saying that contributions are related to beliefs is qualitatively equivalent to asserting that contributions in both treatments hinge on the other's behavior in the previous

\footnotetext{
${ }^{23}$ We excluded from the regression the amount most recently contributed by $i$ 's partner because of its correlation with the variable $\operatorname{Exp}_{i}$. Thus, although the other's one-period lagged contribution is significantly correlated with $c_{i}$, if $\operatorname{Exp}_{i}$ is included in the regression it becomes insignificant.
} 
period. This result is in line with earlier studies (see, e.g., Croson 2000, 2007; Gächter and Renner 2006; Neugebauer et al. 2008), and provides evidence for conditionally cooperative behavior. The coefficient of Info is negative and significant, i.e. (as already summarized by Result 3) participants in II contribute less than participants in PI. Finally, in line with Result 5, the regression supports the absence of a time effect in both treatments, with the exception of period 10 where contributions decline significantly. The next result sums up the most interesting evidence from the regression analysis:

Result 7 In both information conditions, higher contributions are positively related to more optimistic beliefs about the partner's contributions.

To recapitulate the main findings of this subsection, the degree of information has no impact on behavior during the game. Yet, imperfect information leads subjects to contribute less at the outset of the game. The measured risk preference parameters may provide clues as to the nature of this difference.

\subsection{Relationship between risk attitude estimates and contribution behavior}

To investigate how risk preference parameter estimates relate to contributions and whether imperfect information affects such relationship, Table 8 documents the results of two OLS regressions. The dependent variable is each individual's average contribution over all 10 periods in Model 1, and each individual's contribution in period 1 in Model 2. Independent variables are an individual's Pratt risk aversion coefficient $\rho$ (as reported in Tables 2 and 3), the treatment dummy Info (being 0 in $P I$ and 1 in $I I$ ), and the interaction between them.

Both models suggest a significantly negative relationship between contributions and the calculated values of $\rho$ : subjects with a larger $\rho$ tend to contribute less both on average and in the first period. Furthermore, Model 2-comprising period 1 only-shows a clear interaction effect between risk parameters and information conditions: the coefficient of Pratt's $\rho \times$ Info is negative and significant at the 5\%-level, indicating that subjects who appear more risk-averse exhibit lower first-period contributions when information is imperfect. Finally,

Table 8 Relationship between contributions and estimated risk parameter $\rho$ (OLS regression)

\begin{tabular}{|c|c|c|c|c|c|c|}
\hline & \multicolumn{6}{|c|}{ Dependent variable: contributions } \\
\hline & \multicolumn{3}{|c|}{ Model 1: Average } & \multicolumn{3}{|c|}{ Model 2: Period 1} \\
\hline & Estimate & Std. error & $p$-value & Estimate & Std. error & $p$-value \\
\hline Constant & 98.12 & 8.15 & 0.000 & 92.50 & 9.30 & 0.000 \\
\hline Pratt's $\rho$ & -388.80 & 130.06 & 0.004 & -427.80 & 148.40 & 0.005 \\
\hline Info & -34.53 & 14.73 & 0.022 & -19.40 & 16.80 & 0.007 \\
\hline Pratt's $\rho \times$ Info & -1076.83 & 740.9 & 0.151 & -1800.6 & 845.60 & 0.037 \\
\hline Observations & 64 & & & 64 & & \\
\hline Adjusted $R^{2}$ & 0.403 & & & 0.329 & & \\
\hline
\end{tabular}


(in line with previous results) the dummy Info is always negative and significant. ${ }^{24}$ The key findings of this analysis are summarized in last result:

Result 8 The estimated risk aversion parameters are negatively correlated with willingness to contribute. There is an interaction between risk characteristics and information conditions and this impacts first-period decisions.

\section{Conclusions}

This paper has provided experimental evidence on two main issues: the impact of imperfect information on voluntary contribution behavior in linear public goods games, and the relationship between measures of risk attitudes and willingness to contribute. To the best of our knowledge, this is the first study to investigate these issues, and hence to combine two hitherto unrelated strands of experimental work (namely, the one on risk preference parameter estimates and the one on voluntary contributions).

The results indicate that, compared to a setting with perfect information, imperfect information about a public good's value significantly decreases cooperation, with average contributions dropping from $78 \%$ to $41 \%$. The basis for this difference is created in the first period: a risky payoff has a negative impact on contributions at the start of the game, and first-period contribution levels appear to be decisive for future contribution decisions. In fact, the time trend of contributions does not differ between the two treatments, with conditional cooperation being the most likely explanation of behavior in each of them. Thus, while on the one hand this study confirms the findings of previous experiments establishing the importance of conditionally cooperative behavior, on the other hand it provides the first evidence of a significant negative effect of imperfect information on initial contributions.

The explanation this paper proposes for the significant behavioral difference detected in the first period relies on people's elicited risk aversion measures. The main result of the analysis is that the larger a subject's risk aversion coefficient, the less she contributes. Moreover, this negative relationship is particularly pronounced in the first period of the imperfect information treatment.

To conclude, the results reported here undermine the often claimed efficacy of the linear voluntary contribution mechanism in one shot games or at the outset of finitely repeated games, and suggest that it should be in the interest of politicians and firms involved in privately financed public projects to ensure that individuals have good information about their marginal benefits. Needless to say, much more work is necessary to assess the effect of risk and uncertainty in other environments where cooperation is an issue.

Acknowledgements We thank participants at the ESA 2007 World Meeting in Rome, seminar participants at Amsterdam, Jena, Bari, and especially Birendra K. Rai and an anonymous referee for their helpful and constructive comments.

Open Access This article is distributed under the terms of the Creative Commons Attribution Noncommercial License which permits any noncommercial use, distribution, and reproduction in any medium, provided the original author(s) and source are credited.

\footnotetext{
${ }^{24}$ Given that contributions are strongly positively related to beliefs, both in period 1 (see Result 6) and overall periods (see Result 7), the findings of Table 8 do not qualitatively change using expected rather than actual contributions as the response variable. As emphasized earlier, our experimental design abstains from disentangling whether the measured risk parameters affect first beliefs and then contributions or vice versa (i.e., first own predisposition to cooperate and then, due to the false consensus hypothesis, beliefs).
} 


\section{References}

Bagnoli, M., \& Lipman, B. L. (1989). Provision of public goods: fully implementing the core through private contributions. Review of Economic Studies, 56, 583-602.

Berg, J. E., Dickhaut, J. W., \& McCabe, K. (2005). Risk preference instability across institutions: a dilemma. Proceedings of the National Academy of Sciences, 102, 4209-4214.

Brennan, G., González, L., Güth, W., \& Levati, M. V. (2008). Attitudes toward private and collective risk in individual and strategic choice situations. Journal of Economic Behavior \& Organization, 67, 253-262.

Coricelli, G., Fehr, D., \& Fellner, G. (2004). Partner selection in public goods experiments. Journal of Conflict Resolution, 48, 356-378.

Croson, R. (2000). Thinking like a game theorist: factors affecting the frequency of equilibrium play. Journal of Economic Behavior \& Organization, 41, 299-314.

Croson, R. (2007). Theories of altruism and reciprocity: evidence from linear public goods games. Economic Inquiry, 45, 199-216.

Cubitt, R., Starmer, C., \& Sugden, R. (1998). On the validity of random lottery incentive mechanism. Experimental Economics, 1, 115-132.

Dawes, R. (1980). Social dilemmas. Annual Review of Psychology, 31, 169-193.

Di Mauro, C., \& Maffioletti, A. (2004). Attitudes to risk and attitudes to uncertainty: experimental evidence. Applied Economics, 36, 357-372.

Dohmen, T., Falk, A., Huffman, D., Schupp, J., Sunde, U., \& Wagner, G. G. (2006). Individual risk attitudes: new evidence from a large, representative, experimentally-validated survey (C.E.P.R. Discussion Paper No. 5517). London.

Fischbacher, U. (2007). Zurich toolbox for readymade economic experiments. Experimental Economics, 10, 171-178.

Fischbacher, U., Gächter, S., \& Fehr, E. (2001). Are people conditionally cooperative? Evidence from a public goods experiment. Economics Letters, 71, 397-404.

Fischbacher, U., \& Gächter, S. (2006). Heterogeneous social preferences and the dynamics of free riding in public goods (Working Paper No. 261). Institute for Empirical Research in Economics, University of Zurich.

Gächter, S., \& Renner, E. (2006). The effects of (incentivized) belief elicitation in public good experiments (CeDEx Discussion Paper No. 2006-16). University of Nottingham.

González, L., González-Farías, G., \& Levati, M. V. (2005). Logit estimation of conditional cooperation in a repeated public goods experiment (Discussion Papers on Strategic Interaction No. 05-2005). Max Planck Institute of Economics, Jena.

Gradstein, M., Nitzan, S., \& Slutsky, S. (1994). Neutrality and the private provision of public goods with incomplete information. Economics Letters, 46, 69-75.

Hey, J. D., \& Orme, C. (1994). Investigating generalizations of expected utility theory using experimental data. Econometrica, 62, 1291-1326.

Hey, J. D., Morone, A., \& Schmidt, U. (2006). Noise and bias in eliciting preferences (Mimeo). University of Bari.

Holt, C. A. (1986). Preference reversals and the independence axiom. American Economic Review, 76, 508515.

Holt, C. A., \& Laury, S. K. (2002). Risk aversion and incentive effects. American Economic Review, 92, 1644-1655.

Isaac, R. M., \& James, D. (2000). Just who are you calling risk averse? Journal of Risk and Uncertainty, 20, $177-187$.

Isaac, R. M., Walker, J. M., \& Thomas, S. H. (1984). Divergent evidence on free riding: an experimental examination of possible explanations. Public Choice, 43, 113-149.

James, D. (2007). Stability of risk preference parameter estimates within the Becker-DeGroot-Marschak procedure. Experimental Economics, 10, 123-141.

Kachelmeier, S. J., \& Shehata, M. (1992). Examining risk preferences under high monetary incentives: experimental evidence from the people's republic of China. American Economic Review, 82, 1120-1141.

Kachelmeier, S. J., \& Shehata, M. (1994). Examining risk preferences under high monetary incentives: reply. American Economic Review, 84, 1105-1106.

Karni, E., \& Safra, Z. (1987). Preference reversal and the observability of preferences by experimental methods. Econometrica, 55, 675-685.

Kelley, H., \& Stahelski, A. (1970). Social interaction basis of cooperators' and competitors' beliefs about others. Journal of Personality and Social Psychology, 16, 190-197.

Keser, C., \& van Winden, F. (2000). Conditional cooperation and voluntary contributions to public goods. Scandinavian Journal of Economics, 102, 23-39. 
Ledyard, J. O., (1995). Public goods: a survey of experimental research. In J. H. Kagel, \& A. E. Roth (Eds.), The handbook of experimental economics (Chap. 2, pp. 111-194). Princeton: Princeton University Press.

Levati, M. V., \& Neugebauer, T. (2004). An application of the English clock market mechanism to public goods games. Experimental Economics, 7, 153-169.

Marks, M., \& Croson, R. (1999). The effect of incomplete information in a threshold public goods game. Public Choice, 99, 103-118.

Menezes, F. M., Monteiro, P. K., \& Temimi, A. (2001). Private provision of discrete public goods with incomplete information. Journal of Mathematical Economics, 35, 493-514.

Morone, A., \& Schmidt, U. (2008). An experimental investigation of alternatives to expected utility using pricing data. Economics Bulletin, 4, 1-12.

Neugebauer, T., Perote, J., Schmidt, U., \& Loos, M. (2008). Self-biased conditional cooperation: on the decline of cooperation in repeated public goods experiments. Journal of Economic Psychology.

Pratt, J. W. (1964). Risk aversion in the small and in the large. Econometrica, 32, 122-136.

Rabin, M., \& Thaler, R. H. (2001). Anomalies. Risk aversion. Journal of Economic Perspectives, 15, 219232.

Rapoport, A., \& Chammah, A. M. (1965). Prisoner's dilemma: a study in conflict and cooperation. Ann Arbor: University of Michigan Press.

Shogren, J., Shin, S., Hayes, D., \& Kliebenstein, J. (1994). Resolving differences in willingness to pay and willingness to accept. American Economic Review, 84, 255-270.

Shupp, R., \& Williams, A. (2008). Risk preference differentials of small groups and individuals. Economic Journal, 118, 258-283.

Staffiero, G. (2006). Helping the meaner, hurting the nicer: the contribution versus distribution game (IESE Research Papers No. 652). University of Navarra, Spain.

van Dijk, E., \& Grodzka, M. (1992). The influence of endowments asymmetry and information level on the contribution to a public-step good. Journal of Economic Psychology, 13, 329-342.

van Dijk, E., Wilke, H., Wilke, M., \& Metman, L. (1999). What information do we use in social dilemmas? Environmental uncertainty and the employment of coordination rules. Journal of Experimental Social Psychology, 35, 109-135.

Wakker, P. P. (2008). Explaining the characteristics of the power (CRRA) utility family. Health Economics, uncorrected proof published online in www.interscience.wiley.com.

Walker, J., \& Halloran, M. (2004). Rewards and sanctions and the provision of public goods in one shot settings. Experimental Economics, 7, 235-247. 